\title{
Research on Spray Cooling Within Finite Volume Based on Eulerian-Lagrangian Method
}

\author{
Guo He*, Xiaochuan Wang \\ College of Naval Architecture and Power, Naval University of Engineering \\ 717 Jiefang Street, Wuhan, Hubei, 430033, P. R. China \\ heguo953760@163.com
}

\begin{abstract}
Keywords: exhaust system; water-collecting box (WCB); spray cooling; Computational Fluid Dynamics (CFD)
\end{abstract}

\begin{abstract}
The two cooling styles of spraying cooling and water jacket cooling were studied for exhaust gas of engine within water-collecting box (WCB). Based on Eulerian-Lagrangian method, the heat and mass transfer between exhaust gas and water droplets were solved. By comparing the general characteristic index $K / \Delta P$ of six models with different tubes, the model with bended tubes is defined as the best model in which the length of outer tube is less than inner one. With the increase of water spraying flux, the gas temperature and the evaporation rate of water droplets tend to decrease. The decrease of gas temperature can be restrained by the phenomenon of "water assembled", after the spraying flux rise to a certain value with continued increase. Compared with the water jacket cooling, the spraying cooling has a high heat transfer coefficient and a low cooling water cost, as well as reduces the resistance lost of exhaust gas.
\end{abstract}

\section{Introduction}

During the engine of submarine exhaust underwater, the exhaust outlet is below the sea level when reverse flow of the sea water can enter the exhaust pipeline and even the engine [1]. The water-collecting box can prevent the reverse flow of sea water into the pipeline, and can expel the sea water from the exhaust pipe to ensure the safety run of the engine. When the exhaust gas washes the WCB which has double shells, the temperature of the gas will fall. Because the gas flow is separated into branches by sheaves of tubes, the cooling water can cool the hot gas [2]. However, because the WCB is the same as the shell-and tube heat exchanger, the efficiency of heat exchanger is too low to obtain the cooled exhaust, the cost of double shells style is high the monolayer style of WCB.

In this paper, a new type WCB with water spray is raised in which several nozzles are installed on the wall of WCB in the cross section. After the pure cool water was atomized into the WCB, the heat energy of exhaust gas was absorbed by the water which can exert latent heat [3-5], thus the temperature reduced to a great extent.

\section{Mathematical Modeling}

In the mixed stream of exhaust gas and cool water droplets, the exhaust gas and the droplets were treated as continuous phase and discrete phase respectively. Eulerian-Lagrangian method was used to solve the gas-droplets multiphase problem.

Equations of Gas Phase. The exhaust gas was treated as incompressible gas. The conservation equations were described by Reynolds time-average equations. There were plenty of eddies and turbulence airflow, RNG $k^{-} \varepsilon$ turbulent model was used to full the equations. The conservation, turbulent and species equations were expressed in the following general form [6-7].

$$
\frac{\partial\left(\rho_{1} u_{i} \varphi\right)}{\partial x_{i}}=\frac{\partial}{\partial x_{i}}\left(\Gamma \frac{\partial \varphi}{\partial x_{i}}\right)+S_{\varphi}+S_{p \varphi}
$$


where the symbol $\varphi$ stands for any of the following variables: 1) velocity components $u_{i}$ in the $i$ th direction; 2) mass fractions of species $\left.m_{i} ; 3\right)$ enthalpy $h$; 4) turbulence kinetic energy $k$; and 5) turbulence dissipation rate $\varepsilon$. The definitions of $u_{i}$ and $\Gamma$ represent the gas velocity and the diffusion coefficient respectively. $S_{\varphi}$ and $S_{p \varphi}$ stand for the source term and the term which was produced by the interaction between the gas and the droplet phase.

The physical parameters of exhaust gas are different between before and after cooling, such as density and unit heat capacity. If the parameters were treated as constant, the error was large than the normal value, so in the calculation the parameters were modificatory values which vary with the gas temperature.

Equations of Droplet Phase. The droplet was supposed as global, and the vapor as ideal gas. The resistance and gravity which affect the droplets were considered, but other forces were ignored. The movement equations of droplet phase was,

$$
\frac{d\left(m_{p} \boldsymbol{u}_{p}\right)}{d t}=\frac{1}{2} \rho_{1} C_{D} \frac{A_{p}}{4}\left|\boldsymbol{u}_{\boldsymbol{1}}-\boldsymbol{u}_{p}\right|\left(\boldsymbol{u}_{\boldsymbol{1}}-\boldsymbol{u}_{\boldsymbol{p}}\right)+m_{p} \boldsymbol{g}
$$

where $\boldsymbol{u}_{1}$ and $\boldsymbol{u}_{p}$ stand for the vector of gas and droplets velocity. $A_{p}$ represents the surface area of droplet. $C_{D}$ represents the resistant coefficient.

The droplet accord with the law of heat, evaporation and boil in the calculation, thus the three stages can all be described from liquid state to vapor state. When the gas temperature was between 423 and $723 \mathrm{~K}$, the droplets $\left(d_{p}<200 \mu \mathrm{m}\right)$ would vapor rapidly after sprayed into the exhaust gas. The heat radiate of hot gas was ignored [8]. The energy that the droplets absorbed was used for increasing the temperature there selves and exerting the latent heat. The whole equation of energy conservation can be described as follows.

$$
m_{p} c_{p, p} \frac{d T_{p}}{d t}=A_{p} k\left(T_{1}-T_{p}\right)+\frac{d m_{p}}{d t} \gamma
$$

where $c_{p, p}$ stands the unit heat capacity of droplet. The symbol of $k$ and $\gamma$ represent the surface heat transfer coefficient of droplet and the latent heat after evaporating.

The varying equation of droplet diameter is as follow, which can express the evaporation rate of droplet [9].

$$
\frac{d\left(d_{p}^{2}\right)}{d t}=-\frac{4 \lambda}{c_{p, 1}} N u \ln (1+B)
$$

where $c_{p, 1}$ stands for the unit heat capacity of exhaust gas. $D_{p}$ is the droplet diameter. $N u$ is the Nusselt number, and $B$ is the exchange coefficient. $\lambda$ is the heat exchange coefficient of droplet surface.

\section{Numerical Calculation and Analysis}

System Description. The structure of WCB is given in the Fig.1. Different arrows are used to distinguish cool water, exhaust gas and drainage obviously. Atomize nozzles are installed after the tubes outlet seen from the inlet of the tubes. Exhaust gas and cool water flow are described as follow. When exhaust gas enter the WCB, due to the block of the clapboard, gas must enter the sheaves of tubes. Then the gas circumgyrates in the outlet chamber, and leaves the WCB from the exhaust gas outlet. Because of the 90 degree turn of tubes, the direction of airflow is changed from running parallel to encircling the axis [2]. The cool water flows in the interlining from the water inlet at the bottom to the water outlet on the top. Sheaves of tubes are installed in two circles on the clapboard as showed in Fig.2. The bended and folded turnings are two types for the end of tubes. The length of tube is defined as $\mathrm{L}_{0}$. The length of the inner and outer tube is defined $\mathrm{L}_{1}$ and $\mathrm{L}_{2}$ respectively. Different structures of WCB models with different length of tubes and different turnings are listed in the Table 1. 


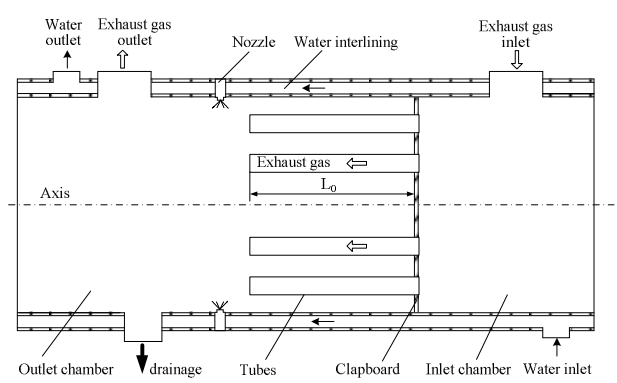

Fig. 1 The sketch map of water-collecting box

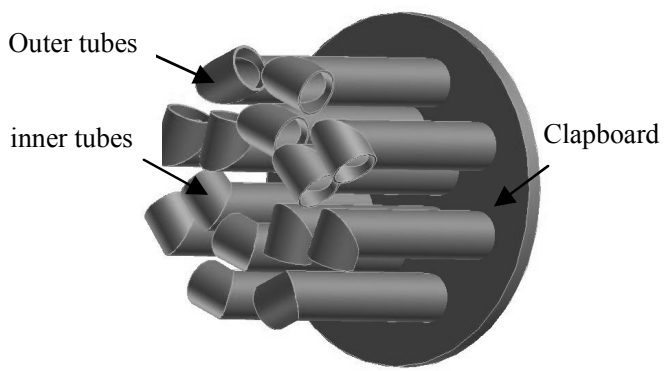

Fig.2 Sketch of clapboard and sheaves of tubes

The $1 / 4$ hydraulic atomizing spray nozzle was used in the experiment, which was produced by Spraying System Company. The orifice diameter of nozzle is $0.71 \mathrm{~mm}$, and the droplets atomized were mist spray. The nozzle worked effectively between $0.2 \mathrm{MPa}$ and $7 \mathrm{MPa}$, and the droplets diameter varied with the atomizing pressure.

Tab.1 Different structures of WCB models

\begin{tabular}{cccl}
\hline Model & $\mathrm{L}_{1}$ & $\mathrm{~L}_{2}$ & $\begin{array}{l}\text { Style of } \\
\text { turning }\end{array}$ \\
\hline I & $\mathrm{L}_{0}$ & $\mathrm{~L}_{0} / 2$ & Folded tube \\
II & $\mathrm{L}_{0} / 2$ & $\mathrm{~L}_{0}$ & Folded tube \\
III & $\mathrm{L}_{0}$ & $\mathrm{~L}_{0}$ & Folded tube \\
IV & $\mathrm{L}_{0}$ & $\mathrm{~L}_{0} / 2$ & Bended tube \\
V & $\mathrm{L}_{0} / 2$ & $\mathrm{~L}_{0}$ & Bended tube \\
VI & $\mathrm{L}_{0}$ & $\mathrm{~L}_{0}$ & Bended tube \\
\hline
\end{tabular}

Tab.2 Four working conditions in simulation

\begin{tabular}{ccc}
\hline $\begin{array}{c}\text { Working } \\
\text { condition }\end{array}$ & $T_{\text {in }} / \mathrm{K}$ & $V_{\text {in }} / \mathrm{m} \cdot \mathrm{s}^{-1}$ \\
\hline 1 & 473 & 10 \\
2 & 573 & 15 \\
3 & 673 & 20 \\
4 & 723 & 25 \\
\hline
\end{tabular}

The Discrete Phase Model (DPM) was used to solve the problem in the FLUENT code. Because there was plenty of turbulence of exhaust gas, the influence of turbulent dispersion on droplets was considered. In Random Path Model, random method was used to consider the influence of turbulent velocity on the droplets paths to describe the running paths exactly. Owing to the great difference of temperature and density between gas and droplets, heat and mass transfer took place between the two phases. Particle Source in Cell Method [10] was used to solve the interaction of the two phases. The droplets reflected after colliding with the solid wall [11]. The four working conditions of gas inlet temperature and velocity in the paper are listed in the Table 2.

Optimize Structure of WCB. The collocation of tubes makes a great infection on the cooling and resistant characteristic of WCB. In the model with equal inner and outer tube length, airflow collided with surface of other tubes, thus exhaust resistance increased. The ratio of total heat transfer coefficient $K$ and pressure drop $\Delta P$ was defined as integrative index to compare characteristics of different models [12]. Fig. 3 shows the index varies with the Reynolds number $(R e)$ of gas inlet. In the same resistance, the bigger index accounts for the better heat transfer effect. It can be shown that with the increase of $R e$, the index decrease. This is mainly because the resistance of duct increases rapidly. In the same working condition, the characteristics of model with bended tubes are better than the one with folded tubes. The characteristics of model with unequal length of outer and inner tubes are better than the equal ones, because the stagger collocation of tube end reduce the flowing resistance. The characteristics of IV model are best of the six ones, there are two main reasons. First, the resistance of bended pipe is lower than the folded one. Second, the shortening of outer tubes extends the time that airflow twists in the container, so the heat transfer effect is enhanced. 


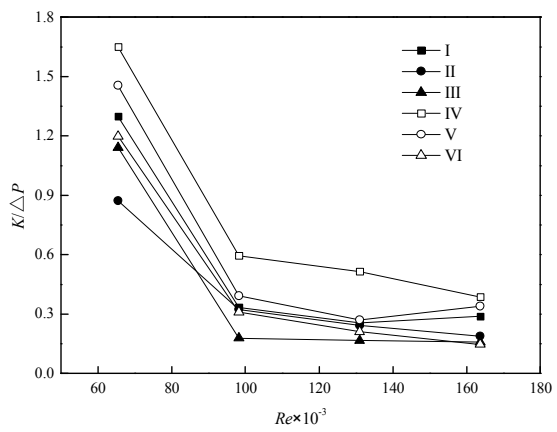

Fig.3 Integrated characteristic index of WCB varies with the Reynolds number

Influence of Spraying Flux. Based on the IV model, define the cool water flux is zero. In the model above, the cool water was sprayed into contact with hot exhaust gas which released heat energy exerted the latent heat of liquid water. Therefore, the gas temperature increased largely. Fig. 4 shows the law of gas outlet temperature and droplets' evaporation rate varied with spraying flux. The experiment data was published in reference [5]. It can be shown that the predicting results agree well with the experimental results. With the increase of spraying flux, the gas outlet temperature and droplets' evaporation tend to fall. When the spraying flux reaches to $0.048 \mathrm{~kg} / \mathrm{s}$, gas temperature can drop to $486 \mathrm{~K}$, while the evaporation is only $80 \%$. Due to the huge difference of initial velocity between gas and droplets, the droplets run along with the gas in the beginning. After period of time, some droplets evaporate, and continue to run along with the gas. However, other droplets that cannot evaporate will attach to the inner wall of WCB. These droplets get together to form liquid water which can be called "water assembled". After the evaporation reach to $80 \%$, the gas temperature drop will be limited, at the same time the "water assembled" will be more serious.

Compare Two Cooling Styles. Better cooling effect can be obtained by spraying little cool water into the WCB. In the same IV model, the water flux by tube-in-tube style of making gas temperature drop $90 \mathrm{~K}$ is 860 times than by spraying. Fig. 5 shows the influence of spraying flux on pressure drop. The pressure drop reduces gradually till to $280 \mathrm{~Pa}$ with the increase of spraying flux. In tube-in-tube style, the pressure drop is $960.2 \mathrm{~Pa}$ which can decrease about $67 \%$ after spraying cool water. It can be found that spraying cooling for WCB has the advantage of enhancing the heat transfer efficiency and reducing gas resistance than the traditional tube-in-tube cooling style. In the WCB with spray cooling and single shell, heat transfer coefficient is higher and resistance is lower than the traditional double shell structure. The structure of the former is simpler than the latter as well.

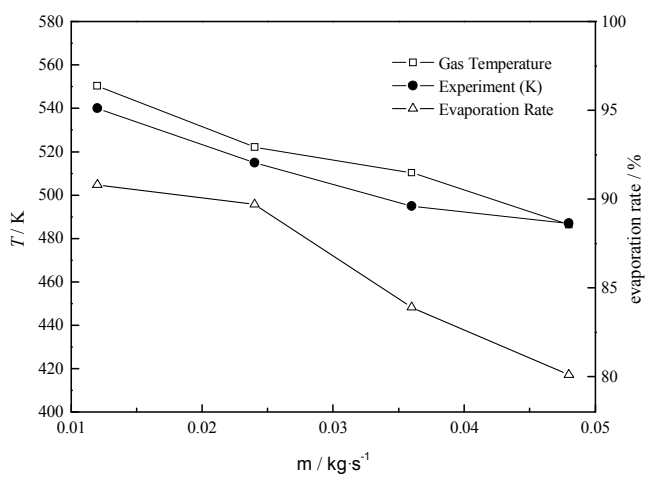

Fig.4 Outlet gas temperature varies with the spraying flux

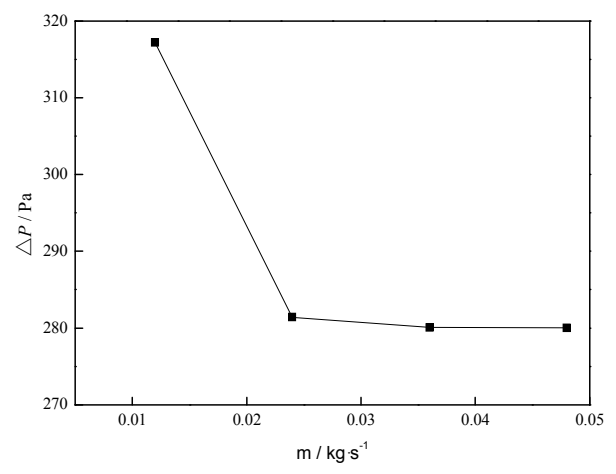

Fig. 5 Gas pressure loss varies with the spraying flux 


\section{Summary}

An Eulerian-Lagrangian method is adopted to simulate the water spray in high temperature gas flow. The heat and mass transfer are considered in the model, and based which different WCB models with different tubes are calculated. The simulation of spraying cooling into IV model is done, and the numerical results agree well with the experimental results in Ref. [5]. The resistance of model with bended tubes is lower than the ones with folded tubes. The general characteristics of IV model are best than other 5 models. When the spray flux reaches to a certain value, the phenomenon "water assembled" is serious. A continuous increase of spray flux does not reduce the gas temperature any more. Spraying cooling has the advantage than the traditional tube-in-tube style, and reduces the resistance as well.

\section{References}

[1] G. He, J. Y. Pu. Function analysis of the water-collecting box used in conventional submarine diesel exhaust system [J]. Journal of Naval University of Engineering, 1998, 10(1): 85-87.

[2] X. C. Wang, G. He and P. Zhou. Influence of tubes on flow and temperature fields within water-collecting box [C] // Proceedings of 2011 International Conference on Mechatronic Science, Electric Engineering and Computer. Jilin: 2011: 1415-1418.

[3] L. He. Study on the design and experiment of spray silencer [D]. Harbin: Harbin Engineering University, 2006.

[4] G. Aguilar, B. Majaron, W. Verkruysse, et al. Theoretical and experimental analysis of droplet diameter, temperature, and evaporation rate evolution in cryogenic sprays $[\mathrm{J}]$. International Journal of Heat and Mass Transfer, 2001, 44(17): 3201-3211.

[5] J. T. Yuan, L. Yang, R. X. Jin, et al. Experimental study on water mist evaporative cooling in power machine exhaust system [J]. Journal of Engineering Thermophysics, 2010, 31(3):461-464.

[6] M. D. Rosello, J. R. Serrano, et al. Analytic-numerical approach to flow calculation in intake and exhaust systems of internal combustion engines [J]. Mathematical and Computer Modelling, 2002, 36(1):33-45.

[7] Y. C. Liu, L. H. Cao, Z. H. Yang, et al. Theoretical research on gas-liquid two-phase flow at outlet of refrigerator capillary [J]. J Cent South Univ: Science and technology, 2007, 38(3): 450-453.

[8] C. C. Tseng, R. Viskanta. Enhancement of water droplet evaporation by radiation absorption [J]. Fire Safety Journal, 2006, 41(3): 236-247.

[9] B. F. Zuo, E. Van, D. Bulck. Fuel oil evaporation in swirl hot gas streams [J]. International Journal of Heat and Mass Transfer, 1998, 41(12):1807-1820.

[10]C. T. Crowe. Modelling spray-air contact in spray-drying systems [C]// Advances in Drying, New York: 1980.

[11]G. Castanet, P. Lavieille, M. Lebouché, et al. Measurement of the temperature distribution within monodisperse combusting droplets in linear streams using two-color laser-induced fluorescence [J]. Experiments in Fluids, 2003, 35(6):563-571.

[12]W. G. Yan, X. L. Yu, G. D. Lu, et al. Heat transfer and pressure drop of heat pipe heat exchanger [J]. Journal of ZHejiang University: Engineering Science, 2011, 45(1): 132-135. 